\title{
Radiative Collapse in Plasma Focus Operated with Heavy Noble Gases
}

\author{
M. Akel · S. Lee
}

(C) Springer Science+Business Media, LLC 2012

\begin{abstract}
Numerical experiments were carried out using 5-phase Lee model on different plasma focus devices with different filling gases. The relation of radiation power gain/ loss terms as a function of temperature has been demonstrated. Variation of radial trajectories versus pressure with 0.1 Torr step has been investigated. Radiative collapse phenomena in plasma focus devices have been observed for heavy noble gases (Ar, $\mathrm{Kr}, \mathrm{Xe})$. Obtained results showed that the line radiation emission and tube voltage have huge values near the radiative collapse regime.
\end{abstract}

Keywords Lee model - Radiative collapse .

Different gases

\section{Introduction}

The Plasma Focus has wide-ranging application potential due to its intense radiation of soft X-ray, hard X-ray, electron and ion beams and fusion neutrons [1] when operated in Deuterium. The use of gases such as $\mathrm{Ne}, \mathrm{Ar}, \mathrm{Kr}$ and Xe for generation of specific SXR or EUV lines for micro-lithography applications [2-4] has been widely discussed in the literature as has the use of nitrogen and

\footnotetext{
M. Akel ( $\square)$

Department of Physics, Atomic Energy Commission, Damascus, P. O. Box 6091, Syria

e-mail: pscientific@aec.org.sy

S. Lee

Institute for Plasma Focus Studies, 32 Oakpark Drive,

Chadstone, VIC 3148, Australia

S. Lee

INTI International University College, 71800 Nilai, Malaysia
}

oxygen to generate the lines suitable for water-window microscopy [5]. Recently argon has been considered for micro-machining due to the harder characteristic line radiation [6]. Various gases including $\mathrm{Kr}$ have been discussed and used for fusion neutron yield enhancement due arguably to mechanisms such as thermodynamically enhanced pinch compressions. Information obtained from the Lee model includes axial and radial velocities and dynamics [7], dimensions and duration of the focus pinch, gross information of temperatures and densities within the pinch, soft X-ray emission characteristics and yield [8-10]. The Lee model in its two-phase form was developed in 1984. Radiation-coupled dynamics was included in the gradually improved five-phase code leading to numerical experiments on radiation cooling [11]. The vital role of a finite small disturbance speed discussed by Potter in a Z-pinch situation [12] was incorporated together with real gas thermodynamics and radiation-yield terms. Plasma self-absorption was included in 2007 [7] improving soft $\mathrm{X}$-ray yield simulation in neon, argon and xenon among other gases [7]. The Pease-Braginskii (P-B) current [13] is known to be that current flowing in a hydrogen pinch which is just large enough for the Bremsstrahlung to balance Joule heating. It is known that in gases emitting strongly in line radiation, the radiation-cooled threshold current is considerably lowered. Lee et al. [14] showed that the equations of the Lee model code [7] may be used to compute this lowering. The code also shows the effect of radiation cooling when operated in the relevant regimes. It is suggested that the neutron enhancement effect of seeding could at least in part be due to the enhanced compression caused by radiation cooling [15]. Ali et al. [16], reported that the effect of self absorption becomes significant when plasma is dense enough to behave as optically thick. It is essential to account for the effect of self-absorption in that 
case. The effect of self absorption of line radiation is investigated in argon plasma by observing the influence of pressure variation of gas. On comparison of the results of numerical experiment considering both aspects i.e. by including and excluding the self absorption term in Lee code an obvious deviation between the trajectories is observed in the 200-300 ns of the slow compression phase in a $3 \mathrm{~kJ}$ plasma focus. Results with self absorption showed that the pinch undergoes slow and very gradual compression. Without self absorption a much more severe compression was observed.

In this paper, the 5-phase Lee Model is used in numerical experiments for different plasma focus devices with different gases to investigate the effect of pressure variations on radial trajectories including the self absorption of line radiation and to study the radiative collapse effect in the plasma focus.

\section{Theoretical Background}

The Pease-Braginskii current $[13,15]$ is the value of current (1.6 MA) at which the Bremsstrahlung radiation (considered as a loss from the plasma) equals the Joule heating of the plasma pinch column in hydrogen assuming Spitzer resistivity. When pinch current exceeds this value, the Bremsstrahlung losses exceed Joule heating and the plasma pinch begins to experience radiative cooling effects at progressively higher currents, until in severe cases, radiative collapse may be observed. The $\mathrm{P}-\mathrm{B}$ current only considers Bremsstrahlung, since at the high temperatures experienced in the hydrogen or deuterium pinch, the gases are fully ionized and there is no line radiation. For gases such as neon, argon, krypton and xenon, there may still be line radiation even at the high pinch temperatures. This line radiation may considerably exceed the effect of Bremsstrahlung in terms of radiation cooling. In that case, the effect of radiation cooling, and eventually radiative collapse may be exacerbated; and may occur at much lower currents. In other words the 'Pease-Braginskii' or threshold current, for these heavier gases may be much lower than the threshold (or P-B) current applied to hydrogen [14].
We consider the following power respectively Bremsstrahlung, Recombination, Line radiation and Joule heating, generated in a plasma column of radius $a_{\text {min }}$, length $Z_{\text {max }}$ at temperature $T$ :

$$
\begin{aligned}
& \frac{\mathrm{d} Q_{\mathrm{B}}}{\mathrm{d} t}=-1.6 \times 10^{-40} N_{i}^{2} Z_{\mathrm{eff}}^{3}\left(\pi a_{\mathrm{min}}^{2}\right) Z_{\mathrm{max}} T^{0.5} \\
& \frac{\mathrm{d} Q_{\mathrm{rec}}}{\mathrm{d} t}=-5.92 \times 10^{-35} N_{i}^{2} Z_{\mathrm{eff}}^{5}\left(\pi a_{\mathrm{min}}^{2}\right) Z_{\mathrm{max}} / T^{0.5} \\
& \frac{\mathrm{d} Q_{\mathrm{L}}}{\mathrm{d} t}=-4.6 \times 10^{-31} N_{i}^{2} Z_{\mathrm{eff}} Z_{n}^{4}\left(\pi a_{\mathrm{min}}^{2}\right) Z_{\max } / T \\
& \frac{\mathrm{d} Q_{\mathrm{J}}}{\mathrm{d} t}=1300 \cdot \frac{Z_{\mathrm{eff}} \cdot Z_{\mathrm{max}}}{\pi a_{\text {min }}^{2}} \cdot I^{2} \cdot T^{-\frac{3}{2}}
\end{aligned}
$$

where, number density $N_{i}$, effective charge number $Z_{\text {eff }}$, atomic number of gas $Z_{\mathrm{n}}$, pinch radius $a_{\text {min }}$, pinch length $Z_{\max }$, plasma temperature $T$ and circuit current $I$. This generated energy is then reduced by the plasma selfabsorption which depends primarily on density and temperature; the reduced quantity of energy is then emitted as the soft X-ray yield.

Several numerical experiments have been carried out using the 5-phase Lee Model code which includes the effect of plasma self absorption; for various plasma focus devices with different filling gases (see Table 1).

To start the numerical experiments, a measured discharge current trace of each device is selected, then the computed total discharge current waveform is fitted to the measured by varying model parameters $f_{m}, f_{c}, f_{m r}$ and $f_{c r}$ one by one until the computed waveform agrees with the measured waveform. First, the axial model factors $f_{m}, f_{c}$ are adjusted (fitted) until the computed rising slope of the total current trace and the rounding off of the peak current as well as the peak current itself are in reasonable (typically good) fit with the measured total current trace. Then we proceed to adjust (fit) the radial phase model factors $f_{m r}$ and $f_{c r}$ until the computed slope and depth of the dip agree with the measured. These fitted values of the model parameters are then used for the computation of all the discharges at various pressures, where the pressure is smoothly varied, with observation of radial trajectories variation versus pressures till radiative collapse were obtained.

Table 1 Tube and electrical parameters for various plasma focus devices

\begin{tabular}{lllllllrr}
\hline Device (ref.) & $E(\mathrm{~kJ})$ & $a(\mathrm{~cm})$ & $b(\mathrm{~cm})$ & $Z_{0}(\mathrm{~cm})$ & $L_{0}(\mathrm{nH})$ & $C_{0}(\mu \mathrm{F})$ & $I_{\text {peak }}(\mathrm{kA})$ & $V_{0}(\mathrm{kV})$ \\
\hline AECS PF-1 [17] & 2.8 & 0.95 & 3.2 & 16 & 1,430 & 25 & 47 & 15 \\
AECS PF-2 [18] & 2.8 & 0.95 & 3.2 & 16 & 200 & 110 & 30 & 115 \\
UNU/ICTP PF [19] & 2.94 & 0.95 & 3.2 & 16 & $4-8$ & 15 & 28 & 370 \\
NX2 [20] & 1.7 & 1.9 & 4.1 & 430 & 11 \\
PF-1000 [21] & 1,064 & 11.55 & 16 & 60 & 33.5 & 1,332 & 2,300 \\
\hline
\end{tabular}




\section{Numerical Experiments: Results and Discussions}

\section{Influence of Line Radiation on the Threshold Current}

To see the relative magnitudes of these power terms as a function of temperature $\mathrm{T}$, we plot the magnitudes of these terms in Fig. 1. We note from Fig. 1 that in the range of plasma focus operation (typically left of $1 \mathrm{keV}$ ) recombination radiation power is much less than line radiation power. Hence in the following analysis we will not consider recombination power. For the radiation power terms [15] we take Bremsstrahlung as the reference so that we compare line radiation power with Bremsstrahlung power. By using the Bennett distribution a relationship between plasma temperature $\mathrm{T}$ and circuit current $\mathrm{I}$ can be obtained [15]. By omitting line radiation term, Lee et al. using Lee model, checked a value of Pease-Braginskii current $I_{\mathrm{P}-\mathrm{B}}$ and showed that for hydrogen $Z_{\text {eff }}=1$; The Pease-Braginskii current $I_{\mathrm{P}-\mathrm{B}}$ computes correctly to a value of 1.6 MA. Considering the general case with all three power terms (Line, Bremsstrahlung and Joule heating), then the line radiation greatly reduces the threshold current $I_{\mathrm{P}-\mathrm{B}}$ [15].

We note from Fig. 1 that in plasma focus operation in a heavy noble gas, typically say in the range $100-1,000 \mathrm{eV}$

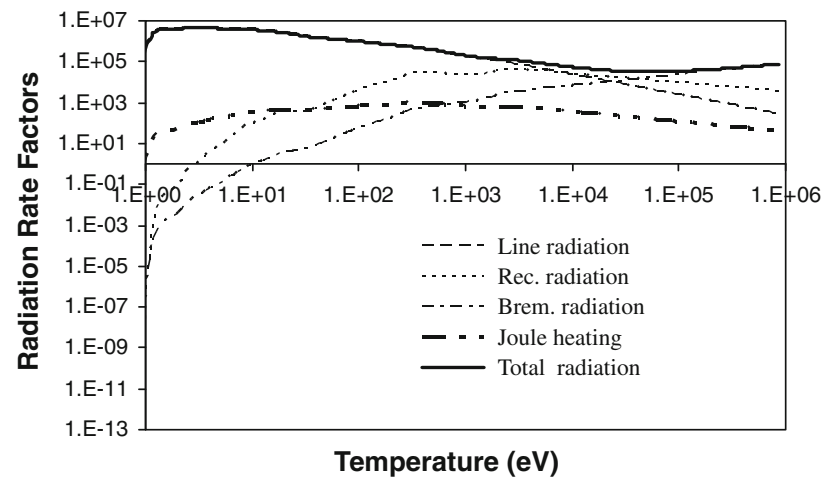

Fig. 1 Showing the relative importance of power gain/loss terms as a function of temperature. Plasma focus operation is at temperatures lower than $10^{7} \mathrm{~K}$ or left of $\log (10) T=3.00$ in the above graph. The example here is computed for krypton for 1 Torr with an anode radius ' $a$ ' of $1 \mathrm{~cm}$

Table 2 Parameters of interested plasma focus devices: $L_{0}, C_{0}, r_{0}$ are inductance, capacitance, and resistance of the capacitor bank, respectively; $V_{0}$-tube voltage, Axial phase: $f_{m}$ (mass swept up the ratio of line power to Bremsstrahlung power has the range 15,000 times to 100 times for krypton plasma, and range 1,000 times to 10 times for xenon plasma [15]; so the threshold current is reduced from $I_{\mathrm{P}-\mathrm{B}}$ by a factor of $\sim 30$ at $100 \mathrm{eV}$ to $\sim 3$ times at $1,000 \mathrm{eV}$; i. e. to $\sim 53 \mathrm{kA}$ at $100 \mathrm{eV}$ to $\sim 533 \mathrm{kA}$ at $1,000 \mathrm{eV}$. In other words at the lower temperature end of plasma focus operation in a gas like $\mathrm{Ar}, \mathrm{Kr}$ or $\mathrm{Xe}$ a current of $50 \mathrm{kA}$ may be enough to reach the threshold at which line radiation begins to exceed joule heating. We also note that the above consideration has not taken into account the effect of plasma selfabsorption. Taking that into consideration the emission power will be reduced. In other words, plasma self absorption will raise the threshold current. We have included radiation-coupled dynamics as well as plasma self-absorption into the Lee Model code. Results with self absorption showed that the compression is slow; while without self absorption radiative collapse was observed due to radiative cooling. Due to self absorption the absorbed radiation kept the plasma from radiative collapse turning the results to be more realistic [16]. Thus the code is able to within its modeling compute the amount of radiation emitted, inclusive of plasma self absorption effects, incorporating these effects into the plasma dynamics.

\section{Radiative Collapse Phenomena}

Numerical experiments have been investigated on plasma focus device parameters to study radiative collapse phenomena. For this purpose, several numerical experiments have been carried out with different gases.

To start the numerical experiments we configure the Lee model code (version RADPF5.15 K) to operate as the plasma focus device and then the fitting procedures of the experimental and numerical current traces reported in the recent publication [22] have been used. To obtain a reasonably good fit for different gases the tabulated parameters are used (see Table 2). With these parameters, the computed total current trace agrees reasonably well with the experimental trace for studied devices. These fitted values of the model parameters are then used for the computation of all the discharges at various pressures.

factor), $f_{c}$ (plasma current factor); Radial phase: $f_{m r}$ (mass swept up factor), $f_{c r}$ (plasma current factor)

\begin{tabular}{lclllllll}
\hline Device (ref.) & $L_{0}(\mathrm{nH})$ & $C_{0}(\mu \mathrm{F})$ & $r_{0}(\mathrm{~m} \Omega)$ & $V_{0}(\mathrm{kV})$ & Gas & $f_{m}$ & $f_{c}$ & $f_{m r}$ \\
\hline ICTP PFF [23] & 110 & 30 & 12 & 13.5 & Argon & 0.044 & 0.7 & 0.1 \\
AECS PF-2 [24] & 270 & 25 & 35 & 15 & Argon & 0.05 & 0.7 & 0.15 \\
AECS PF-2 [25] & 280 & 25 & 25 & 15 & Neon & 0.1 & 0.7 & 0.2 \\
AECS PF-1 [26] & 1,600 & 25 & 80 & 15 & Nitrogen & 0.1 & 0.7 \\
\hline
\end{tabular}




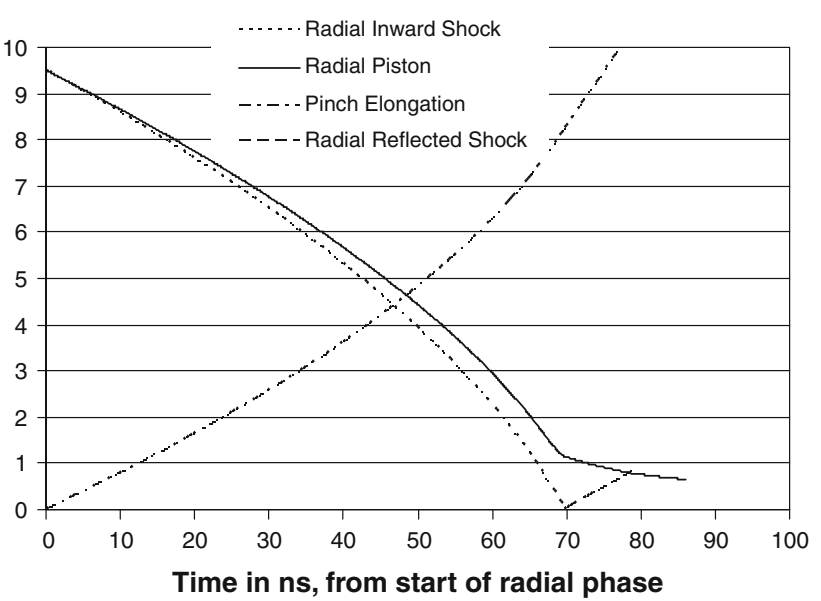

Fig. 2 Radial dynamics on AECS PF-2 plasma focus with argon filling gas at 0.4 Torr

Radial trajectories variations were observed to find the radiative collapse with varying pressures. We start with argon plasma focus, so the code was executed after configuring the required device. The radial trajectories were plotted against time at various pressures ranging from 0.1 to 1.9 Torr with a step of 0.1 Torr. Figure 2 acts as a reference situation and shows the radial dynamics at 0.4125 Torr for AECS PF-2 device. The following Figs. 3, 4 show variations of radial trajectories versus pressures on AECS PF-2 and UNU/ICTP PFF devices. It is clear from these Figures that radiative cooling reduces the pinch radius as pressure is increased, above 0.8 Torr, where strong radiative collapse is evident in the range 0.85-1.5 Torr for AECS PF-2 (Fig. 3), while for UNU/ ICTP PFF the strong radiative collapse is evident in the range above 2 Torr (Fig. 4). For AECS PF-2, the radial inward shock wave starts at $9.49 \mathrm{~mm}$ and is driven to the axis after $70 \mathrm{~ns}$ with the driving magnetic piston trailing it by some $1.1 \mathrm{~mm}$ as the shock front hits reaches the axis. A

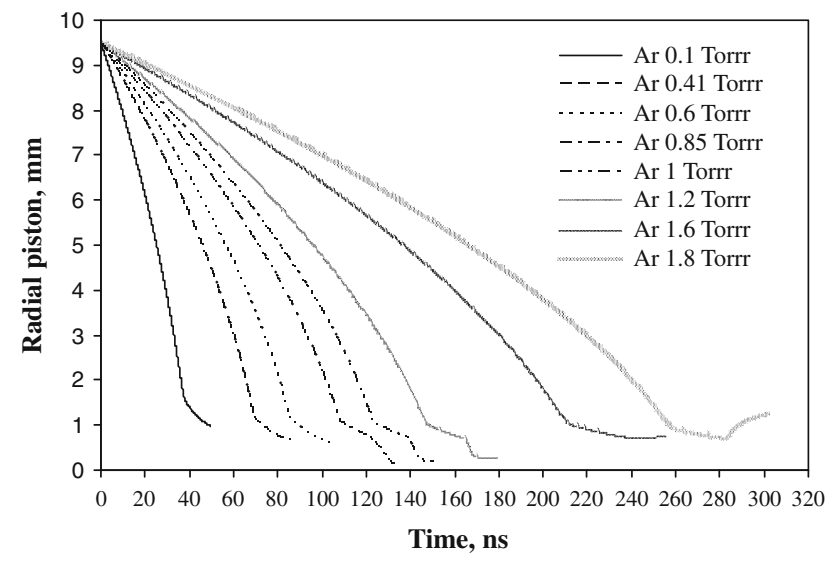

Fig. 3 Variations of radial trajectories on AECS PF-2 versus argon pressure

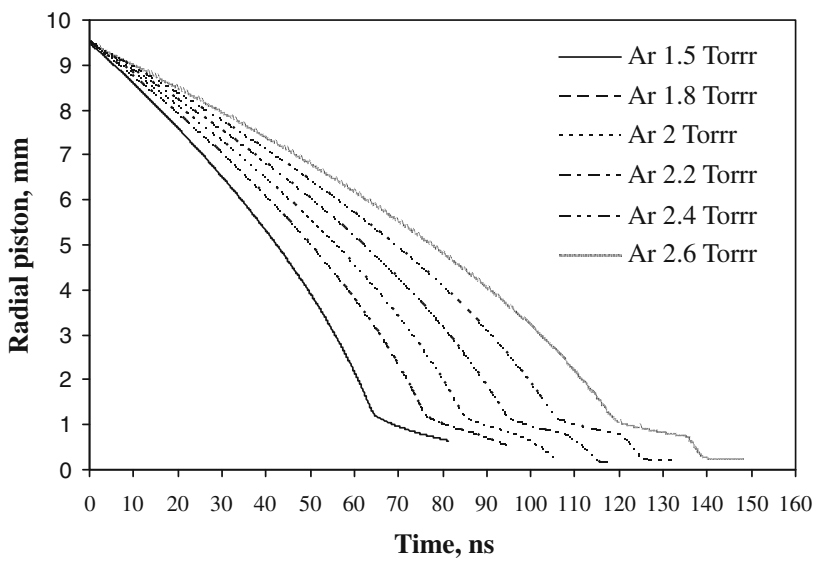

Fig. 4 Variations of radial trajectories on UNU/ICTP PFF versus argon pressure

reflected shock (RS) goes outwards and after some 8 ns hits the incoming piston. At this time the pinch starts and the column compresses inwards a little, as is typical Computed data indicates that even in this shot the radiation power emitted (mostly line) already exceeds the Joule heating power with the pinch temperature in this shot reaching $300 \mathrm{keV}$. This means that there is already net power loss (or radiation cooling) but the radiation cooling is insufficient to perceptibly affect the dynamics at the pinching power provided by the available pinch current at that time. At 0.85 Torr and a pinch temperature of $190 \mathrm{eV}$ with a pinch current of just under $66 \mathrm{kA}$, radiative collapse is obvious with the radius collapsing in a few ns to the cut-off radius of $0.1 \mathrm{~mm}$ set in the model. At 1 Torr with a pinch temperature of $154 \mathrm{eV}$, the collapse does not reach the cutoff radius. In this case the model maintains a small radius $(0.2 \mathrm{~mm})$ pinch for about $5 \mathrm{~ns}$. These are the conditions (high density, relatively long pinch duration $12.2 \mathrm{~ns}$ ) which produce huge line yields of more than $22.5 \mathrm{~J}$. At 1.2 Torr and pinch temperature of $130 \mathrm{eV}$ with a pinch current of just $57 \mathrm{kA}$, the speeds are much slower now and the pinch occurs late so the current has dropped considerably reducing the pinching force; the radiative collapsed radius is now bigger $(0.3 \mathrm{~mm})$. Finally at $1.7-2$ Torr, the column blows out instead of pinching in as the RS hits the piston.

For krypton plasma focus, to start the numerical experiments, we select the following model parameters $f_{m}=0.04, f_{c}=0.7, f_{m r}=0.1, f_{c r}=0.7$. Then many numerical experiments using Lee model have been investigated for wide pressure range with step 0.1 Torr for $L_{0}=270 \mathrm{nH}, C_{0}=25 \mu \mathrm{F}, r_{0}=32 \mathrm{~m} \Omega$ and $V_{0}=15 \mathrm{kV}$. These experiments have shown that, the radiative collapse starts at 0.44 Torr, with the pinch temperature of $453 \mathrm{eV}$ and with a pinch current of just $75 \mathrm{kA}$. With pressure increasing the pinch temperature and the pinch current decreases and the radiative collapse becomes stronger and 


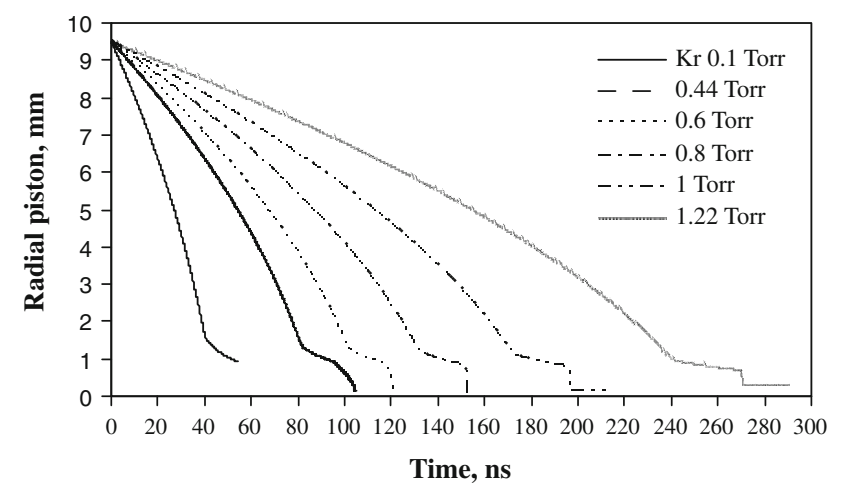

Fig. 5 Variations of radial trajectories versus Krypton pressure

continues for a very small period about $1 \mathrm{~ns}$ after a slow compression phase. At 1 Torr with a pinch temperature of $154 \mathrm{eV}$, with a pinch current of just under $62 \mathrm{kA}$ the collapse does not reach the cut-off radius directly after starting of compression phase. In this case the model maintains a small radius $(0.15 \mathrm{~mm})$ pinch for about $12 \mathrm{~ns}$. These are the conditions (high density $61 \times 10^{23} \mathrm{~m}^{-3}$, relatively long pinch duration $16 \mathrm{~ns}$ ) which produce huge line yields of more than $110 \mathrm{~J}$. At 1.22 Torr and pinch temperature of $77 \mathrm{eV}$ with a pinch current of just $40 \mathrm{kA}$, the radiative collapse occurs with pinch radius $0.3 \mathrm{~mm}$ for few of nanoseconds. Finally at 1.7-2 Torr, the column blows out instead of pinching in as the RS hits the piston. It is noticed that with increasing pressures a focused effect will be delayed (see Fig. 5).

For xenon plasma focus, to start the numerical experiments, we select the following model parameters $f_{m}=0.05, f_{c}=0.7, f_{m r}=0.1, f_{c r}=0.7$. Then many numerical experiments using Lee model have been also investigated for wide pressure range with step 0.1 Torr for $L_{0}=270 \mathrm{nH}, C_{0}=25 \mu \mathrm{F}, r_{0}=32 \mathrm{~m} \Omega$ and $V_{0}=15 \mathrm{kV}$. These experiments have shown that, the radiative collapse starts at 0.2 Torr, with the pinch temperature of $770 \mathrm{eV}$ and with a pinch current of just $76 \mathrm{kA}$. With pressure increasing until 0.6 Torr the pinch temperature and the pinch current decreasing and the radiative collapse becomes stronger and continues for a very small period about $1 \mathrm{~ns}$ after a slow compression phase. At 0.63 Torr with a pinch temperature of $68 \mathrm{eV}$, with a pinch current of just under $40 \mathrm{kA}$ the collapse does reach the cut-off radius directly after starting of compression phase. In this case the model maintains a small radius $(0.15 \mathrm{~mm})$ pinch for about $12 \mathrm{~ns}$. These are the conditions (high density $61 \times 10^{23} \mathrm{~m}^{-3}$, relatively long pinch duration $20 \mathrm{~ns}$ ) which produce huge line yields of more than $140 \mathrm{~J}$ (Fig. 4). This pressure is the upper limit for Lee model operating (Fig. 6). From these numerical experiments, it is noticed that tube voltage has a high maximum value of $100 \mathrm{kV}$, corresponding to a maximum value of ion density about

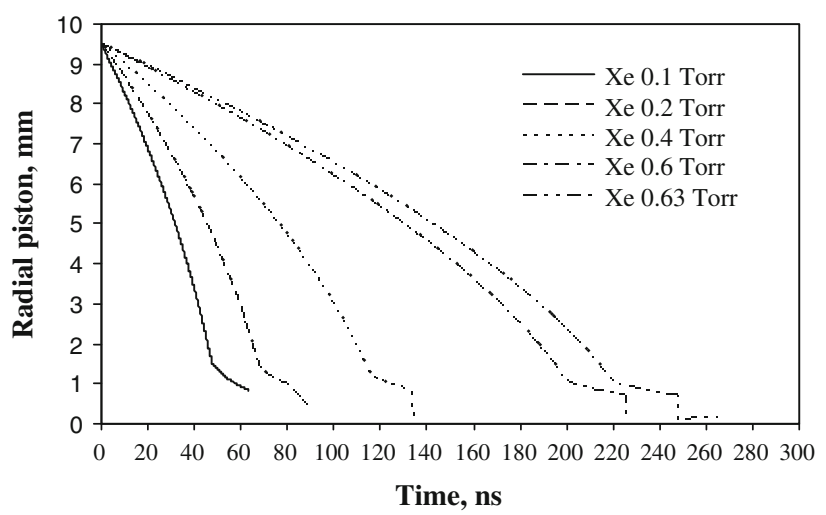

Fig. 6 Variations of radial trajectories versus Xenon pressure

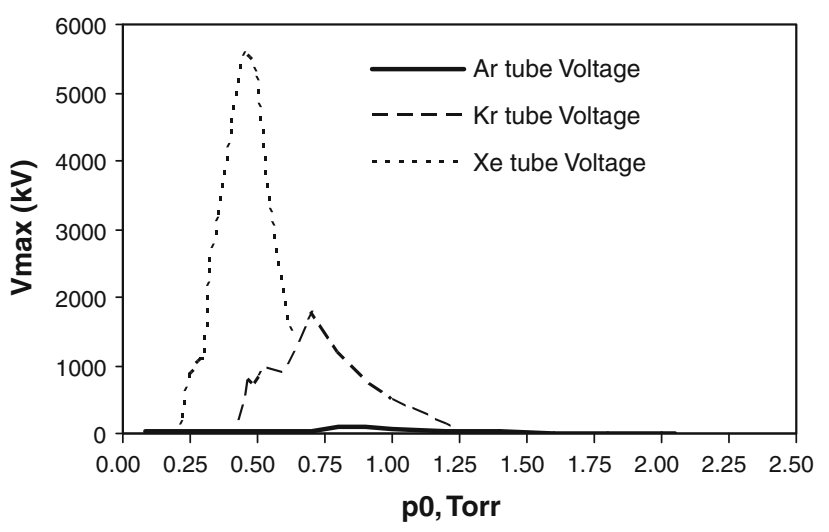

Fig. 7 Tube voltage variations versus gas pressures

$61 \times 10^{23} \mathrm{~m}^{-3}$ at 0.85 Torr for plasma focus operated with Argon gas. And even higher tube voltage values of 1,800 and $5,600 \mathrm{kV}$ at lower pressures down to 0.7 and 0.4 Torr for Krypton and Xenon plasma focus, respectively, (see Fig. 7). In addition to that, many sets of numerical experiments using Lee model on parameters of different plasma focus devices (NX2, PF1000) were carried out. Radiative collapse phenomena have been also observed at higher pressure with heavy noble gases. From numerical experiments with neon, oxygen and nitrogen plasma focus, it can be said that no radiative collapse phenomena has been noticed under a wide pressure range. Finally, based on obtained results by five phase Lee model, we can say that type and pressure of the plasma focus play an important role in radiative collapse creation. This phenomenon produces an extreme increase in tube voltage and generates huge line radiations in the plasma focus.

\section{Conclusions}

Numerical experiments showed that at the lower temperature end of plasma focus operation in a gas like Argon, 
Krypton or Xenon a current of $50 \mathrm{kA}$ may be enough to reach the threshold at which line radiation begins to exceed joule heating. The five phase Lee model code was run for different plasma focus devices with various gases like $\mathrm{Ar}$, $\mathrm{Kr}$ and $\mathrm{Xe}$ and demonstrates radiative cooling leading to radiative collapse at a pinch current ranging from $50 \mathrm{TO}$ $100 \mathrm{kA}$. A huge line radiation from plasma focus has been found at suitable high pressures. The creation of the consequential extreme conditions of density and pulsed power is of interest for research and applications.

Acknowledgments The authors would like to thank Director General of AECS, for encouragement and permanent support.

\section{References}

1. A. Bernard, H. Bruzzone, P. Choi, H. Chuaqui, V. Gribkov, J. Herrera, K. Hirano, A. Krejci, S. Lee, C. Luo, F. Mezzetti, M. Sadowski, H. Schmidt, K. Ware, C.S. Wong, V. Zoita, Scientific status of plasma focus research. Mosc. J. Phys. Soc. 8, 93-170 (1998)

2. R.R. Prasad, M. Krishnan et al., Neon dense plasma focus point $\mathrm{X}$-ray source for $<0.25 \mu \mathrm{m}$ lithography. SPIE 2194, 120-128 (1994)

3. S. Lee, P. Lee, G. Zhang, X. Feng, V.A. Gribkov, M. Liu, A. Serban, T. Wong, High rep rate high performance plasma focus as a powerful radiation source. IEEE Trans. Plasma Sci. 26(4), 1119-1126 (1998)

4. K. Bergmann, O. Rosier, W. Neff, R. Lebert, Pinch-plasma radiation source for extreme-ultraviolet lithography with a kilohertz repetition frequency. Appl. Opt. 39(22), 3833-3837 (2000)

5. R. Lebert, W. Neff, D. Rothweiler, Pinch plasma source for X-ray microscopy with nanosecond exposure time. J. X-Ray Sci. Technol. 6, 2 (1996)

6. V.A. Gribkov, A. Srivastava, P.L.C. Keat, V. Kudryashov, S. Lee, Operation of NX2 dense plasma focus device with argon filling as a possible radiation source for micro-machining. Plasma Sci. IEEE Trans. 30, 1331-1338 (2002)

7. S. Lee, Radiative dense plasma focus computation package: RADPF (2012), http://www.plasmafocus.net. http://www.plasma focus.net/IPFS/modelpackage/File1RADPF.htm

8. S. Lee, Plasma focus model yielding trajectory and structure, in Radiations in Plasmas, vol. II, ed. by B. McNamara (World Scientific, Singapore, 1984), pp. 978-987

9. T.Y. Tou, S. Lee, K.H. Kwek, Non perturbing plasma focus measurements in the run down phase. IEEE Trans. Plasma Sci. 17(2), 311-315 (1989)
10. S. Lee, A sequential plasma focus. IEEE Trans. Plasma Sci. 19(5) (1991)

11. J. Ali, Development and studies of a small plasma focus. Ph.D. dissertation (Universiti Teknologi Malaysia, Malaysia, 1990)

12. S. Lee, A. Serban, Dimensions and lifetime of the plasma focus pinch. IEEE Trans. Plasma Sci. 24(3), 1101-1105 (1996)

13. K.N. Koshelev, V.I. Krauz, N.G. Reshetniak, R.G. Salukvadze, Yu.V. Sidelnikov, E.Yu. Khautiev, The formation of the micropinch structure in plasma focus by the addition of heavy impurities. J. Phys. D Appl. Phys. 21, 1827 (1988)

14. S. Lee, S. H. Saw, J. Ali, Numerical experiments on radiative cooling and collapse in plasma focus operated in krypton. J. Fusion Energ. (2012) doi:10.1007/s10894-012-9522-8

15. S. Lee, S. H. Saw, Multi-radiation modelling of the plasma focus, in Fifth International Conference on Fronties of Plasma Physics and Technology, 18-22 April 2011, Singapore

16. Z. Ali, S. Lee, F.D. Ismail, Saktioto, J. Ali, P.P. Yupapin, Radiation self absorption effect in Ar gas NX2 mather type plasma focus. Phys. Proced. 8, 393-400 (2011)

17. Sh. Al-Hawat, Axial velocity measurement of current sheath in a plasma focus device using a magnetic probe. IEEE Trans. Plasma Sci. 32(2), 764-769 (2004)

18. Sh. Al-Hawat, M. Akel, C.S. Wong, X-ray emission from argon plasma focus contaminated with copper impurities in AECS PF-2 using five channel diode spectrometer. J. Fusion Energ. 30(6), 503-508 (2011)

19. S.H. Saw, P.C.K. Lee, R.S. Rawat, S. Lee, Optimizing UNU/ ICTP PFF plasma focus for neon soft X-ray operation. IEEE Trans. Plasma Sci. 37(7), 1276-1282 (July 2009)

20. S. Lee, S.H. Saw, P.C.K. Lee, R.S. Rawat, Numerical experiments on plasma focus neon soft X-ray scaling. Plasma Phys. Control. Fusion 51, 105013 (2009)

21. S. Lee, S.H. Saw, P.C.K. Lee, R.S. Rawat, H. Schmidt, Computing plasma focus pinch current from total current measurement. Appl. Phys. Lett. 92(11), 111, 501, (March 2008)

22. Sh. Al-Hawat, M. Akel, S. Lee, S.H. Saw, Model parameters vs. gas pressure in two different plasma focus devices operated in Argon and Neon. J. Fusion Energ. 31(1), 3-20 (2012)

23. M Akel, Yield optimization of Helium and Lyman emissions in low energy plasma focus operated with argon. J Fusion Energ. (2011). doi:10.1007/s10894-011-9499-8

24. M. Akel, S. Lee, Practical optimization of AECS PF-2 plasma focus device for argon soft X-ray operation. J. Fusion Energ. 31(2), 122-129 (2012)

25. S. Al-Hawat, M. Akel, S. Lee, Numerical experiments on neon soft X-ray optimization of AECS-PF2 plasma focus device. J Fusion Energ. 30(6), 494-502 (2011)

26. M. Akel, Sh. Al-Hawat, S. Lee, Numerical experiments on soft $\mathrm{X}$-ray emission optimization of nitrogen plasma in $3 \mathrm{~kJ}$ plasma focus SY-1 using modified Lee model. J. Fusion Energ. 28(4), 355-363 (2009) 